\title{
Establishment of Geodetic Network in Nigeria
}

\author{
Ikharo I. Blessing ${ }^{1}$, Matthew N. Ono², Olaniyi Saheed S. ${ }^{3}$ \\ ${ }_{1,2}$ Nnamdi Azikiwe University, Awka Anambra State, Nigeria \\ ${ }^{3}$ Federal School of Surveying, Oyo, Oyo state Nigeria
}

\begin{abstract}
This paper focus on the establishment of Geodetic Network in Nigeria which serve as the framework and basis for every spatially referenced system in the country. It explains the historical development of Nigeria geodetic network, its mode of establishment, data acquisition method, computation and adjustment principles. The use recent positioning satellites (GNSS) and its several methods were discussed with the Least Squares Adjustment techniques. Literatures on best practices were reviewed to identify gaps that need to be covered so as to have an accurate and efficient geodetic network in the Nigeria. The research is basically on principles and methodology on the establishment of Geodetic Network in Nigeria and silent on hardware and software selection for its establishment.
\end{abstract}

Keywords : Geodetic Network, Geodetic, GNSS, GPS, Reference System, Least Squares Adjustment

\section{INTRODUCTION}

The theoretical framework is based on the knowledge of the concepts of precise geodetic control network, geodetic datum, and specifications of a geodetic controls using Global Positioning System (GPS) technology. To establish a geodetic network, one must have an understanding of the various types of geodetic networks, their design, accuracy requirements, and essence. This chapter gives a brief overview of geodetic control networks; their types, design, accuracy requirements, and importance. A geodetic reference network is the wire-frame or the skeleton on which continuous and consistent mapping, Geographic Information Systems (GIS), and surveys are based. Traditionally, geodetic control points are established as permanent physical monuments placed in the ground and precisely marked, located, and documented. Locating spatial features with respect to geodetic control enables the accuracy assessment of these features. Interest and activity regarding geodetic control has dramatically increased at all government levels because of the need for accurate maps and surveys needed for national development and environmental studies.

The geoid which is regarded as a physical model that approximates the real earth as much as possible is not suitable for mathematical computations. For this reason, the physical earth (geoid) is then related to ellipsoid. [1]

Geodetic networks are usually established to serve as reference framework for mapping, space research, mineral exploration, geodesy and geodynamic studies. They consist of monumented points whose geodetic coordinates are known to be closee to their natural coordinates. [2].

Conventionally, classical (traditional) geodetic networks are separated into horizontal and vertical geodetic networks. The horizontal geodetic, with 
geodetic coordinates ( $\phi$ and $\lambda$ ) are referred to as horizontal geodetic datum, while the vertical geodetic network with orthometric height $(\mathrm{H})$ is referred to the geoid, surface (approximated, by the means seas level over the oceans). Threedimensional geodetic network, with space rectangular coordinates $(\mathrm{X}, \mathrm{Y}, \mathrm{Z})$ or the geodetic coordinates $(\phi, \lambda)$ is the natural way of presenting the network of monumented points on the earth's surface [2]. The relationship between the ellipsoidal height $h$, the orthometric height $\mathrm{H}, \mathrm{N}$ is the geoid undulation and the geoid undulation is:

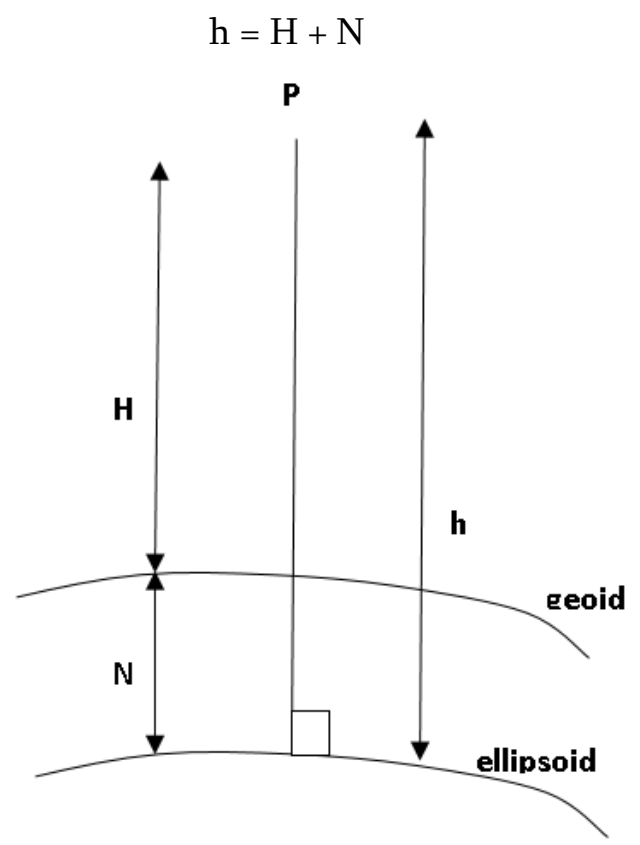

Figure 2.1 : Relationship between Orthometric and Ellipsoidal Heights [3]

Geoid undulation can be determined both by gravimetric surveys and by the inclusion of points of known elevation in GPS surveys. When the average undulation of an area has been determined, the residual undulations over the surveyed area must still be determined [4].

Nowadays, heights of points could be reckoned with respect to an ellipsoid; in fact, we have already introduced this height as the ellipsoidal height, $\mathrm{h}$.
However, this height does not correspond with our intuitive sense of height as a measure of vertical distance with respect to a level surface. Two points with the same ellipsoidal height may be at different levels in the sense that water would flow from one point to the other. Ellipsoidal heights are purely geometric quantities that have no connection to gravity potential; and, it is the gravity potential that determines which way water flows. An unperturbed lake surface comes closest to a physical manifestation of a level surface and mean sea level (often quoted as a reference for heights) is reasonably close to a level surface. We may define a level surface simply as a surface on which the gravity potential is constant. [5] With today's advanced geodetic satellite techniques, in particular GPS, and accurate knowledge of the geoid, one prefers so called global ellipsoids that fit the geoid globally (whose centre of figure is at the centre of mass, and whose axes coincide with the directions of the International Terrestrial Reference Frame (ITRF).

The relationship between the Cartesian coordinates $(\mathrm{X}, \mathrm{Y}, \mathrm{Z})$ and the geodetic coordinates $(\phi, \lambda, \mathrm{h})$ can be expressed. Cartesian coordinates $(\mathrm{X}, \mathrm{Y}, \mathrm{Z})$ can be expressed as a function of the geodetic coordinates $(\phi, \lambda, \mathrm{h})$ by the following equations: Jekeli, (2006)

$$
\begin{array}{ll}
\mathrm{X} & =(\mathrm{V}+\mathrm{h}) \operatorname{Cos} \phi \operatorname{Cos} \boldsymbol{\lambda} \\
\mathrm{Y} & =(\mathrm{V}+\mathrm{h}) \operatorname{Cos} \phi \operatorname{Sin} \\
\mathrm{Z} & =\quad\left\{\left(1-\mathrm{e}^{2}\right) \mathrm{V}+\mathrm{h}\right\} \operatorname{Sin} \phi
\end{array}
$$

The inverse solution, i.e. expressing the triplet $(\phi, \lambda, \mathrm{h})$ as a function of $(\mathrm{X}, \mathrm{Y}, \mathrm{Z})$ involves a nonlinear mathematical relationship. The longitude follows straight forwardly from equations (2.2 and (2.3) as 


$$
\operatorname{Tan} \lambda=\frac{y}{x}
$$

One only needs to pay attention to the quadrant of the longitude. The geodetic latitude $\phi$ follows from the nonlinear equation (2.4) using some iterative technique. For this purpose, it is convenient to rewrite equation (2.4) as

$\tan \phi=\frac{x}{\sqrt{x^{2}+y^{2}}}\left(1+\frac{e^{2}}{x} N \sin \phi\right)$

and use $\phi$ initial $=\tan ^{-1}\left[\frac{x}{\left(1-e^{2}\right) \sqrt{x^{2}+y^{2}}}\right]$

On the right- hand side of equation (2.6) to start the iteration. The iteration stops after successive solutions yield negligible changes in the geodetic latitude. After convergence, the geodetic height follows from

$$
\mathrm{h}=\frac{\sqrt{x^{2}+y^{2}}}{\cos \phi}
$$

as can be readily verified (Leick, 2013). Where e and $\mathrm{N}$ in equations (2.2) - (2.4) are the eccentricity and radius of curvature in the prime vertical, respectively, of the reference ellipsoid.

Hence, the reference ellipsoid is defined as a surface generated by rotating an ellipse 1800 about its minor axis forms the surface called the ellipsoid of revolution. This ellipsoid is the most often used in the geodetic calculations to represent the earth is called a reference ellipsoid or a spheroid. Many international mapping agencies have stopped regarding spheroid and ellipsoids as equivalent because a spheroid is a very complex surface while an ellipsoid is simple to a degree surface.

The general equation of an ellipsoid is

$$
\frac{x^{2}+y^{2}}{a^{2}}+\frac{z^{2}}{b^{2}}
$$

Where $\mathrm{a}$ and $\mathrm{b}$ are the semi-axes of the rotated ellipse, the centre of which is at the origin. Usually in geodesy, this bi-axial ellipsoid of revolution is used to represent the earth's shape and size by the following two parameters, "a" as semi- major axis or equatorial radius, "b" as semi- minor axis or polar radius, or " $\mathrm{f}$ " the flattening where

$$
f=\frac{a-b}{a}
$$

The set of parameters that defines the shape and size, as well as the relationship between this ellipsoid and the real earth (geoid) is called geodetic datum.

There are two types of geodetic datum and global geodetic datum. However, the focus of this research is on global geodetic datum. A global geodetic datum on the other hand adopts a reference ellipsoid approximating the shape of the entire global geoid and having its centre at the earth's centre of gravity. Hence global datums are geocentric datums. The World Geodetic system 1984 (WGS84) "coordinate system" or reference system on which the GPS is based was established through Doppler observations from the TRANSIT satellite system [6]. Global positioning satellite system was developed, based on the World Geodetic system of 1984 (WGS84) ellipsoid. Basically, GPS is a method of position determination by method of trilateration by determining the distances (pseudo ranges) from satellites in space, whose accurate positions (ephemeris) are known in time. Hence, it is also called navigation satellite using Time and Ranging NAVSTAR [6].

Reference ellipsoids used in global datum are the world geodetic system 1984 (WGS84) has $a=$ $6478137.00 \mathrm{~m}, I / f=298.257223563$, and the geodetic reference system 1980 (GRS80) has $a=6378137.00 \mathrm{~m}$, $1 / f=298.257222101$ 
The WGS84 ellipsoid is used by GPS while the GRS80 ellipsoid is used in International Terrestrial Reference Frame (ITRF) coordinate system. However, on the other hand, certain specialized observing systems like the TOPEX satellite altimetry system, have adopted ellipsoid that differ from the standard ones like GRS80 or WGS84. It is, therefore, extremely important that the user of any system of coordinates or measurements, understands what ellipsoid is implied [5].

The concept of geodetic datum derives from the need to replace the complicated realities of the physical earth's surface by a simple well defined and regular geometric figure on which computations and geodetic operations can be conveniently accomplished. An ellipsoid of revolution endued with the normal gravity field of the earth having its centre at the centre of mass of the earth and its axes oriented relative to the conventional average terrestrial system (ATS), provide the required geodetic datum for global purposes. The surface of geodetic figure is referred to as geodetic datum surface [2].

Hence the integration of vertical and horizontal networks into a three- dimensional network is possible and geoid in Nigeria is not determined. Therefore, the integration of the horizontal and vertical geodetic networks into a three- dimensional geodetic network through this method is not achievable for now [6].

[7], observed that the "geodetic datum" is regarded as the set of parameters which define the relationship between a reference ellipsoid and real earth. These usually include semi-major axis (a), flattening (1), or eccentricity (e), deflection of the vertical ( $\xi, \eta)$, geoidal undulation $(\mathrm{N})$, and orientation parameters (e.g. Polar motion and Azimuth).

\subsection{Reference System and Reference Frame}

In order to refer to a point on area on the surface of the Earth, a coordinate system is needed. The most common way of stating terrestrial position is by the use of geographic coordinates (latitude and longitude). They define a point on the surface of an ellipsoid which approximately fits the globe, the third coordinate, ellipsoidal height, is provided by the distance of the point in question to the ellipsoidal surface along a straight line perpendicular to the ellipsoidal surface. Another common coordinate system is the Cartesian coordinate system. In this system, three mutually perpendicular axes unambiguously locate any point. A third type of coordinate system in common use is the Eastings and Northings, also called plane coordinates, grid coordinates or map coordinates. This system uses a two- dimensional Cartesian system in which the two axes are known as Eastings and Northings. This is very useful for visual display purposes [8].

No matter the type of coordinate system used, a suitable origin is required with respect to which the coordinates are stated. For instance, we cannot use Cartesian coordinates unless we have defined an origin point of the coordinate axes and defined the directions of the axes in relation to the Earth whose features we wish to measure. We must also state the parameters of the ellipsoid of the coordinate system.

The coordinates system must now be made available for use in practice. If the coordinate system is going to be used consistently over a wide area, it will involve setting up some infrastructure of points to which users can have access, and the coordinate of which are known at the time of measurement. The network of these reference points with known coordinates is called the coordinate. Terrestrial reference frame (TRF) and its purpose is to realize the coordinate system by providing accessible points of known coordinates. A common example of TRF is the 
networks of national survey triangulation pillars seen on hill tops. Another example is the constellation of 24 GPS satellites operated by the United States Department of Defence. Users can avail themselves of these TRF points by observing with a positioning tool (a Theodolite or a GPS receiver in these examples) and hence obtain new positions of previously unknown points in the coordinate system.

The major modem reference systems include the world geodetic system (WGS), the international terrestrial references system (ITRS) and the parametric Zamli (PZ) [8]. The world geodetic system (WGS) realized by the US department of defence was produced using available surface gravity observations, results, from triangulation and trilateration networks and large amounts of Doppler and optical satellite and employing processing techniques.

The current system, WGS 84 is satellite based. WGS 84 reference frame has undergone some refinements, one in 1994 and another in 1996 designated '(WGS 84 (G730)' and 'WGS 84 ( G873)' where the 'G' indicates that the refinements were obtained through Global positioning system (GPS), techniques and the number following the ' $G$ ' indicates the GPS week number of implementation [8]. This means that all GPS observations are referenced to WGS 84. For all practical purposes, the parameters of the WGS 84 reference ellipsoid are identical to those of the Geodetic Reference System Type equation here.1980 (GRS 80) ellipsoid. They use the same value of $6378137 \mathrm{~m}$ for the semi-major axis and have the same orientation with respect to the centre of mass and the origin of the coordinate system. The centre and origin of the coordinate system are coincident with the earth's centre of mass [8].
These reference systems have their advantages and disadvantages. [12], observed the advantages and disadvantages of the reference systems as follows:

The advantage of a geodetic coordinates system is that; It is inherently immutable. This system was the order of the day before the advent of satellite positioning systems. Its realization was direct and every geodetic coordinate system was really fixed and oriented with respects to the origin of the network for good. Such a system is thus quite transparent to the user since it is understood that the system relates to something that has been optimized for a particular country. For instance, the origin of the coordinate system can be physically seen.

The disadvantage of geodetic coordinate systems is that: It is quite different from continent to continent (empire to empire, country to country, and municipality to municipality). This dealing with positions of points in different geodetic coordinate systems (on different geodetic datum) requires a knowledge of transformation equations and parameters which is generally a fairly complicated matter.

Therefore, it would be a misunderstanding of geocentric coordinate system when GNSS / GPS (satellite) observations are referred to local datum (Minna datum). This could only be possible when there are transformation parameters that could be used to transform the local datum to the geocentric datum. Unfortunately, there is no valid published transformation parameter for Nigeria.

\subsection{Geodetic Networks}

Geodetic networks are designed to serve a wide range of users, ranging from reconnaissance level surveying, through small and large scale mapping, engineering and land surveying, to scientific research, for example in geophysics and the study of plate 
tectonics. In the context of land information systems, the function of such a network is to define a unique reference framework through which all mapping field surveys and other data collection activities in any given area can be spatially integrated. A control network is needed so that the relative positions of different features can be established or compared.

Within a limited area, it is possible to establish a local survey network unconnected to any national system. In a country such as Nigeria this was the practice for many years as the size of the country and the difficulties in communication were so great as to preclude any alternative [9]. A local network provides a number of fixed points to which all surveys, both topographic and cadastral, can be connected so that land parcel boundaries can, for example, be related to the physical objects appearing on the topographic maps. For most land information management purposes, where townships are well separated and where no national geodetic control exists, a local network is adequate. Existing map series can be used as the basic framework. If coordinate values have been established on the local system, they can, at some future date, be transformed to the national system as and when such becomes available. Ideally, however, a national network should be established first, so that all areas in a country can be cross- related. This avoids the inevitable confusion that will otherwise arise when, for every point there are two sets of coordinates, the old and new.

However, with the improvements in positioning accuracy with the evolving technology, many lay users of the technology are creating confusion by using modem reference systems and the local geodetic datum interchangeably. This makes planning of regional and continental geospatial projects exceptionally difficult. It also presents difficulties in the utilization of regional and global geodetic information. As a result of these, the research is aimed at establishing a precise geodetic network for the study area that would be of regional and global applications.

\section{TYPES OF GEODETIC CONTROL}

There are basically three types of geodetic control, namely: Horizontal, Vertical and Gravity. This thesis will focus on horizontal and vertical controls. Gravity network will therefore, not be discussed in this document.

\subsection{Horizontal Control}

In surveying, a horizontal control is defined as a system of points whose horizontal positions and interrelationships have been accurately determined for use as fixed references in positioning and correlating map features [10].

Horizontal control can be established by a number of methods, among the commonest being Triangulation, Trilateration and Precise traversing.

\subsection{Vertical Control}

Vertical control networks are series of points on which precise heights or elevations have been established. Vertical control stations are typically called bench marks. As part of a vertical information network, the bench marks elevation is known relative to a datum usually mean sea level. Normally, the orthometric height is known. The orthometric height is defined as the distance between the vertical reference surface (geoid) and the vertical geodetic station on the surface of the Earth measured along the plumb line between the two. Differential and trigonometric leveling are the commonest methods of determining elevation. [11]. In differential leveling, a sequence of lines-of-sight is established. Two readings are taken along the line of sight, one at a known benchmark elevation and the other at a point 
of unknown elevation. The difference between the two is used to establish the elevation of the unknown point. For geodetic work, three- wire levelling is used [11], [10]. In this method, a levelling instrument with an eyepiece having three separate horizontal lines is used. Elevation is determined by finding the average reading for each of the horizontal lines. Global Positioning System (GPS) can also be used to obtain vertical heights. However, GPS only gives accurate ellipsoidal heights.

\subsection{Ground Marks}

Control networks have two elements: the physical and the mathematical. Traditionally, much time, effort and money have been spent on the construction of triangulation pillars and ground marks that would stand the test of time. [9], suggest that;

A good permanent mark is like an iceberg, has most of its volume below the surface and it presents a pyramidal or rounded upper part to the accidental or malicious shocks it has to resist. It is more important to the certain of finding the marks after 30 years than to be able to find them easily at any time.

The physical maintenance of the fabric of a geodetic network is as important as the maintenance of the records which relate to it. The pillars are usually constructed in accordance with the specification of the country concern. For example, in Nigeria, the specification for international, interstate boundary beacon, primary GPS beacon and primary triangulation beacon is 2.3meters height of reinforced concrete pillar. Beneath each pillar, a brass bolt screwed $0.01 \mathrm{~m}$ into Theodolites holder and sunk $0.1 \mathrm{~m}$ into pillar. A central pipe of $1.2 \mathrm{~m}$, a concrete base of $0.8 \mathrm{~m}$ minimum and lower block of $0.3 \mathrm{~m}$ with a lower centre mark [12]. About $1.1 \mathrm{~m}$ of the pillar is buried leaving $1.2 \mathrm{~m}$ above the ground. The principle of treating survey pillars like icebergs was, in the past, correct. Today there are two practical differences. The first is the availability of the satellite network, which in effect creates beacons in the sky. The second is that relatively cheap earth anchor are available which are light and portable and can be driven firmly into the ground in [9].

\section{GLOBAL NAVIGATION SATELLITE SYSTEM (GNSS)}

Today the Global Navigation Satellite Systems (GNSS) led by the U.S.A. Global positioning system (GPS) and the Russian Global Orbiting Navigation Satellite System (GLONASS) have become the most important systems for satellite geodesy [6].

Worldwide navigation satellite systems have been given the generic name Global Navigation Satellite Systems (GNSS). As the name suggests that GNSS are primarily navigation systems designed to give instantaneous determination of position and velocity anywhere, anytime and in all kinds of weather on or above the earth's surface. Historically, they were conceived for military purposes, but have now become widely in use as the most indispensable satellite technology system for a variety of civilian applications, including geodesy [6], [1].

Many geodesists regard GNSS as the last word for solving existing problems in geodesy. As previously stated, GNSS and especially the Global Positional System (GPS) have become excellent tools beneficially applied to such areas as agriculture, astronomy, aviation, surveying I charting I mapping, civil air Il and I sea navigation, developmental / security constructions, fisheries, the geosciences, meteorology, etc. In different modes of operation, the accuracy ranges from several metres to centimetres and millimetres level. 


\subsection{Global Positioning System (GPS)}

Global positioning system (GPS) is a new and unique approach to surveying which has emerged in recent years. This system, which grew out of the space program, relies upon signals transmitted from satellites, for its operation. It has resulted from research and development paid for by the military to produce a system for global navigation and guidance. With GPS, it is now possible to obtain precise timing and positioning information anywhere on the earth with high reliability and low cost. The system can be operated day or night, rain or shine, and does not require cleared lines of sight between survey stations. This represents a revolutionary departure from conventional surveying procedures which rely on observed angles and distances for determining point positions. GPS has gained worldwide acceptance and the technology is being used for virtually every type of survey. There is little doubt that GPS has affected the practice of surveying more profoundly than any other technology to date in [1].

GPS was originally designed for military guidance and positioning, but has attracted a wide variety of civilian users in the positioning and navigation applications fields. Additional applications have been developed in commercial aviation navigation, boating and shipping navigation, tracking and rail car positioning, emergency routing automobile dashboard, electronic charts and orienteering navigation. Also manufacturers are now installing GPS chips in cell phones to help satisfy the service requirement for precise caller locations in [4]

\subsubsection{GPS Receivers}

GPS receivers range in ability (and cost) from surveylevel (millimeter) receivers capable of use in surveys requiring high accuracy are quite expensive.

The major differences in the receivers are the number of channels available (the number of satellites that can be tracked at a time) and the condition whether or not the receiver can observe both $\mathrm{Ll}$ and $\mathrm{L} 2$ frequencies and measure both code and carrier phases. Generally speaking, dual frequency receivers attract high -cost because they require much shorter observation times for positioning measurements than do the less expensive single-frequency receivers and can be used for realtime positioning. The more expensive receivers can data log every second and can be used in all GPS survey modes with shorter observation times whereas less expensive receivers may data log every 15 seconds and can be restricted to a certain type of survey and will require longer observation times and perhaps longer processing times as well. [4]

\subsection{GPS Position Measurements}

Position measurements generally fall into one of two categories, code measurement and carrier phase measurement; civilian code measurement is presently restricted to the coarse/ Acquisition (C/A) code, which could provide accuracies only in the range of $10-15 \mathrm{~m}$ when used in point positioning, and accuracies in the submetre to $5 \mathrm{~m}$ range when used in various differential positioning techniques. Precision code (which is encrypted and called the $\mathrm{P}(\mathrm{Y})$ code) measurements can apparently provide the military with much better accuracies. Point positioning is the technique that employs one GPS receiver to track satellite code signals, so that it can directly determine the coordinates of the receiver station. [4], [7]

\subsubsection{Static GPS Mode}

Static refers to a fixed observation location. In static GPS mode, the receiver is stationary for the duration of the observation for a specific period, normally not less than one hour for most modem dual frequency GPS receivers. This method is also known as classical static. In a static relative positioning technique, two or more receivers are used to collect GNSS data 
simultaneously. This technique is typically associated with baselines greater than $20 \mathrm{krn}$ and the procedure begins with a base (reference) receiver set up over a known station (whose coordinates are given in WGS 84 or ITRF) while the roving receiver occupies the unknown station. Most static methods produce higher redundancy and a bit of more accuracy than kinematic GPS. Therefore, they are mostly used in surveying control and geodetic networks establishment and baseline vectors for crustal dynamics monitoring in which millimeter or 1.0 to $0.1 \mathrm{ppm}$ accuracies are achieved [3]. For the accuracy requirement of this project, a static relative positioning technique would be adopted.

\subsubsection{Differential GPS}

The theory behind differential positioning is on the use of two or more GPS receivers to track the same satellites simultaneously. Unlike relative positioning, at least one of the GPS receivers must be set up at a station of known coordinates. This base station receiver's satellites signals compute the position, so it can compare its computed position with its known position and derive a difference value. This difference reflects all the measurements errors (except for multipath) for nearby receivers. This "difference" can be included in the nearby roving GPS receivers' computations to remove the common errors in their measurements [4]. Real- time differential surveys; also known as RTK has accuracies from $1-2 \mathrm{~cm}$. Requires a base receiver occupying a known station, which the radio transmits error corrections to any number of roving receivers thus permitting them to perform data gathering and layout surveys in real time. All required software is on board the roving receivers. Dual-frequency receivers permit reinitialization after loss of lock. Baselines are restricted to about $10 \mathrm{~km}$. five satellites are required. This, or similar technique, is without doubt the feature of many engineering surveys.
Differential Positioning; involves obtaining satellite measurements at a known base station in order to correct simultaneous same-satellite measurements made at rover receiving stations.

Corrections can be post processed, or corrections can be real-time (RTK) as when they are broadcast directly to the roving receiver. [1]

\subsection{GPS Carrier Phase Measurement}

GPS codes, which are modulations of the carrier frequencies are comparatively lengthy. Compare the CIA code at $29.3 \mathrm{~m}$ and the $\mathrm{p}$ code at $29.3 \mathrm{~m}$ with the wavelengths of $\mathrm{Ll}$ and $\mathrm{L} 2$ of $0.19 \mathrm{~m}$, and $0.24 \mathrm{~m}$, respectively. It follows that carrier-phase measurement have the potential for much higher accuracies than do code measurements when we observed how electronic distance measurement (EDM) equipment measured distances. EDM distances are determined by measuring the phase delay required to match up the transmitted carrier wave signal with the return signal (two-way signalling).

The equation for distance calculation is given by

$$
L=\frac{\eta \lambda+\phi}{2}
$$

Where $\phi$ is the partial wavelength determined by measuring the phase delay (through comparison with an onboard reference), $\mathrm{n}$ is the number of complete wavelengths (from the EDM to the prism and back to the EDM instrument), and A is the wavelength. The integer number of wavelengths is determined as the EDM instrument successively sends out (and receives back) signals at different frequencies thus permitting the creation of equations allowing for the computation of the unknown [4].

GPS ranging involves only one-way signalling, other techniques must be used to determine the number of 
full lengths. GPS receivers can measure the phase delay (through comparison with onboard carrier replicas) and count the full wavelengths after lock on to the satellite has occurred but more complex treatment is required to determine $N$, the initial cycle ambiguity. That is, $N$ is the number of full wavelengths sent by the satellite prior to lock on. Because a carrier signal is comprised of a continuous transmission of sine-like waves with no distinguishing features, the wave count cannot be accomplished directly.

$$
P=\phi+N \lambda+\text { errors }
$$

Where, $P=$ satellite-receiver range

$\phi=$ measured carrier phase

$\lambda=$ wavelength

$N=$ initial ambiguity (the number of full wavelengths at lock -on [4]

\section{ADJUSTMENT COMPUTATION}

The field reductions must be carried out before the adjustment of the coordinates of the common points established by the terrestrial and satellite observations. Here, an independent network adjustment of satellite and terrestrial observations must be carried out. The precision and reliability of the network and that of satellite network gives the basis for deriving appropriate corrections for the study areas using the appropriate models.

\subsection{Network Design}

Network design includes the determination of the number and location of existing control stations for network constraints, the selection of new project station locations, and the relative dispersion of network observations. Space-based measurement systems, such as GPS, are not significantly affected by such factors as network shape or intervisibility [13]
[14]. This provides the opportunity to focus upon the intent of the project control, and geographically sensitive issues rather than the limitations of the measurement technique. However, network design does have relevance both for the elimination or reduction of potential error sources as well as for providing adequate ties to the existing geodetic reference system. These concerns may be addressed by the choice of which existing control stations should be included, as well as the planning of new station locations and network observation periods [15]. To meet a local network accuracy classification, a GPS project must be connected to sufficiently accurate and well distributed existing control [15]. All of the control stations to which the network will be constrained must have positions known on the same datum and epoch since additional uncertainty in the time-dependent positioning models (TDP), which would need to be added into the network constraints, will likely add unacceptable levels of uncertainty to the network

\subsection{Error Ellipse}

Statistically error ellipse can be defined as the area of constant probability density. In surveying and geodesy terms, error ellipse simply is a graphical representation of the marginal standard deviation of the parameters and the covariance between them. Error ellipses are usually computed during adjustment of horizontal or three - dimensional network. They allow a convenient way for interpretation of the directional station position accuracy. Error ellipses are also used in optimising a network [16]

Error ellipse is an estimate of the precision of the adjusted 2D coordinates at each station, and the relative precision of the $2 \mathrm{D}$ coordinates between stations. Error ellipses are frequently used in connection with two-dimensional networks in order 
to make the directional precision of station location visible [17]. They can be computed for each adjusted station and between connected stations. The stations error ellipses are computed from the aposteriori covariance matrix for each station. To compute the relative error ellipse between stations, the aposteriori covariance matrix for the AT station, the $\mathrm{TO}$ station and the aposteriori covariance matrix which correlates the two stations are used. Because the error ellipse is a two-dimensional statistical result, the 2D expansion factor is used to scale the semi-major and semi-minor axes to the chosen confidence level (in this case, 95\%).

[18] gave the semi-major axis $\sigma_{x^{1}}^{2}$, semi-minor axis $\sigma_{y^{1}}^{2}$ and the orientation of the error ellipse $\theta$ as:

$$
\begin{gathered}
\sigma_{x^{\mathrm{I}}}^{2}=\frac{\sigma_{x}^{2}+\sigma_{y}^{2}}{2}+\left[\frac{\left(\sigma_{x}^{2}-\sigma_{y}^{2}\right)^{2}}{4}\right]+\sigma_{x y}^{2} \\
\sigma_{y^{\mathrm{I}}}^{2}=\frac{\sigma_{x}^{2}+\sigma_{y}^{2}}{2}-\left[\frac{\left(\sigma_{x}^{2}-\sigma_{y}^{2}\right)^{2}}{4}\right]+\sigma_{x y}^{2} \\
\tan 2 \theta=\frac{2 \sigma_{x y}}{\sigma_{x}^{2}-\sigma_{y}^{2}}
\end{gathered}
$$

[18] further pointed out that the variances $\sigma_{x^{1}}^{2}$ and $\sigma_{y^{1}}^{2}$ are the eigenvalues of the covariance matrix of the random vector $\left[\begin{array}{l}X \\ Y\end{array}\right]$.

[18] also stated that the probability of the position given by $\mathrm{X}$ and $\mathrm{Y}$ lies on or within the error ellipse is

$$
\begin{aligned}
P\left[\frac{X^{2}}{\sigma_{x}^{2}}+\frac{Y^{2}}{\sigma_{y}^{2}} \leq c^{2}\right] & =P\left[U \leq c^{2}\right] \\
& =\int_{0}^{c^{2}} \frac{1}{2} e^{-u / 2} d u=1-e^{-c^{2} / 2}
\end{aligned}
$$

Where, $\mathrm{c}=$ Expansion Factor $($ for $95 \%, \mathrm{c}=2.448$ ).

The probability $P\left[U \leq c^{2}\right]$ is represented by the volume under the bivariate normal density surface within the region defined by the error ellipse. The semi major axis and semi minor axis confidence ellipse is determined by

Semi major axis confidence ellipse $=\sigma_{x^{l}} c$

Semi minor axis confidence ellipse $=\sigma_{y^{I}} c$

\subsection{Measures of Accuracy}

Practical considerations require coordinates of points to be selected as the parameters in the model for the adjustment of geodetic networks. The coordinates comprised in the vector $X$ as well as the corresponding variance-covariance matrix $\sum_{X a}$ are not invariant quantities of the model, they depend on the selection of the geodetic datum. The fact that these results are not unique causes some problems in assessing the precision of the adjusted positions of points [19].

[19] also stated that once point estimation is performed, we need to know how much the deviation of the estimate is likely to be from the true value. How good the estimation is and how much it can be relied on should be established. An absolute answer is never possible but probabilities as to how close the estimate is to the true value can be established.

The accuracy information about the estimated parameters is all contained in the $\mathrm{u} \mathrm{x} \mathrm{u}$ variancecovariance matrix, $\sum_{X a}$. 
$\sum_{X a}=\hat{\sigma}_{\circ}^{2} N^{-1}=\hat{\sigma}_{\circ}^{2}\left(\begin{array}{cccc}Q_{11} & Q_{12} & \ldots & Q_{1 n} \\ a_{21} & Q_{22} & \ldots & Q_{2 n} \\ \ldots & \ldots & \ldots & \ldots \\ Q_{n 1} & Q_{n 2} & \ldots & Q_{n n}\end{array}\right)$

The measures of accuracy that may be obtained from the variance-covariance matrix as given by [19] are:

1. Standard deviation of the adjusted coordinates.

2. Confidence intervals.

3 Error ellipse of the adjusted positions.

\subsection{Aposteriori Variance and Aposteriori Standard Error}

The models for the computation of the a posteriori variance and a posteriori standard error as given in [20] are:

1. APosteriori Variance $\left(\hat{\sigma}_{o}^{2}\right)$

$$
\hat{\sigma}_{o}^{2}=\frac{V^{T} W V}{m-n}=\frac{V^{T} W V}{r}
$$

2. APosteriori Standard Error $\left(\hat{\sigma}_{o}\right)$

$$
\hat{\sigma}_{o}=\sqrt{\frac{V^{T} W V}{m-n}}=\sqrt{\frac{V^{T} W V}{r}}
$$

\subsection{Standard Error of the Adjusted Parameters}

[20] gave the model for the computation of the standard error of the adjusted parameters as:

$$
\hat{\sigma}_{x i}=\hat{\sigma} \circ \sqrt{Q_{n n}}=\sqrt{\hat{\sigma}_{o}^{2} Q_{n n}}
$$

Where,

$Q_{n n}$ is a diagonal element of the inverse of the normal matrix $\left(N^{-1}\right)$.

\subsection{Variance-Covariance of the Adjusted}

\section{Observation}

The model for the computation of the variancecovariance of the adjusted observation $\left(\sum_{L a}\right)$ is given as:

$$
\sum_{L a}=A \sum_{X a} A^{T}=\hat{\sigma}_{\circ}^{2} A N^{-1} A^{T}
$$

Semi manor axis confidence ellipse $=\sigma_{y^{l}} c$

\section{REVIEW OF EXISTING RELATED STUDIES}

Nowadays, several hundred geodetic satellites are in orbit, supplemented by a large number of remote sensing satellites and navigation systems like GPS and GLONASS, which will be followed by the European Galileo satellites in 2013. While these developments have made satellite-based geodetic network surveying more flexible and cost effective than its terrestrial equivalent, the continued existence of fixed point networks is still needed for administrative and legal purposes on local and regional scales. Global geodetic networks cannot be defined to be fixed, since geodynamics are continuously changing the position of all continents by 2 to $20 \mathrm{~cm}$ per year. Therefore, modern global networks like ETRF or ITRF show not only coordinates of their "fixed points", but also their annual velocities [21].

Network densification has, until recently, been the only way of making the positions of surveyed points both technically and economically accessible to the users. It is a prerequisite for establishing an integrated survey system. The requirements imposed on points of the integrated survey system by the wide range of users include various position information and point locations, monumentation and spacing. Accuracy requirements include the stringent $5 \mathrm{~cm}$ and $1 \mathrm{~cm}$ (with $400 \mathrm{~m}$ spacing or less) for 1:500 large scale mapping and relocation surveys in urban areas, 
respectively, and the much lower 5-10 m requirement for medium scale mapping at 1:50000 scale.

Network densification provides these requirements in several stages. The major concern is that at each stage in the hierarchy, the densification network can be improperly defined within the existing network. However, since several techniques exist which lead to rigorous solutions this research will examine the techniques and the rigour of the solution in-context of practicality and economy. It embraces three mam areas:

(a) Analysis of rigorous densification schemes including post-adjustment correction considerations, (b) statistical testing of densification networks as solitary networks and in conjunction with the existing networks and (c) possibility of strain analysis applications to quality control of densification networks.

\subsection{The Nigerian Geodetic Network}

The first geodetic surveys of Nigeria were performed by the British Royal Engineers in 1910 - 1912. The existing geodetic networks (horizontal and vertical networks) in Nigeria started to be observed in the late 1920's and most of the network was materialized between the late 1940's and early 1960's [22]. The common reference system in use until recently is the Minna Datum ( $\lambda=9^{\circ} 38^{\prime}$ 09".000; $0=6^{\circ} 30^{\prime}$ 59".000) and the Clarke 1880 ellipsoid (modified) (Mugnier, 2009). However, during the last decades, many geodetic pillars materializing the reference frame have been destroyed, and only a small percentage of beacons are still usable. Furthermore, the original network was implemented using techniques having lower accuracy and requiring the installation of points at locations of difficult access (e.g., top of hills). The Nigerian Geodetic Control Network was observed by terrestrial measurements of angles and distances (bases). The observations took place between the late 1940's and early 1960's. A Triangulation Station at Minna (L40) was selected as the origin of the network. The projection used for the Datum is Clark 1880 (modified) [23].

The geodetic network was established in hierarchical manner as an array of triangles. Observations were carried out in series of chains and categorized as Primary Chain which consists of Primary triangulation points, Secondary Chain which consists of the secondary triangulation points and Tertiary Chain which consists of the tertiary triangulation points. Traverse points were used to augment the network in the southern and northern parts of the country where the topography was not conducive for the establishment of triangulation points due to nonavailability of hills and high ground.

A geodetic network is a network of triangles which are measured exactly by techniques of terrestrial surveying or by satellite geodesy. In "classical geodesy" (up to the sixties) this is done by triangulation, based on measurements of angles and of some spare distances; the precise orientation to the geographic north is achieved through methods of geodetic astronomy. The principal instruments used are theodolites and tacheometers, which nowadays are equipped with infrared distance measuring, data bases, communication systems and partly by satellite links. Electronic distance measurement (EDM) was introduced around 1960, when the prototype instruments became small enough to be used in the field. EDM increased network accuracies up to 1: 1 million $(1 \mathrm{~cm}$ per $10 \mathrm{~km}$; today at least 10 times better), and made surveying less costly. The geodetic use of satellites began around the same time. By using bright satellites like Echo I, Echo II and Pageos, global networks were determined, which later provided support for the theory of plate tectonics. 


\subsection{Review of relevant studies done in Nigeria and other countries}

[24] presented a research work titled "Surveying with GPS, Total Station and Terrestrial Laser Scanner: A Comparative Studies". The objective of the thesis is to evaluate and compare precision, accuracy and time expenditure of Total station (TS), Global Positioning System (GPS), and Terrestrial Laser Scanner (TLS). A GPS receiver measures the incoming phase of the satellite signals to millimetre precision. However, as the satellite signals propagate from satellites to receivers they pass and are affected by the atmosphere. The atmosphere that influences the incoming signal consists of the ionosphere and troposphere. Disturbance in the atmosphere cause degradation in the accuracy of the observations. The GPS surveying is a differential method; a baseline is observed and computed between two receivers. When the two receivers observe the same set of satellites simultaneously, most of the atmospheric effects are cancelled out. The short the baseline is the more these effects will be reduced, as more likely it is that the atmosphere through which the signal passes to the two receivers will be identical. Real time kinematics (RTK) data collection uses differential GPS corrections broadcast by a base receiver to solve for coordinates at a rover receiver in real time. The methodology adopted to accomplish the objective of the research, was the collection of data from field. The researcher used three different surveying instruments for field measurements. The instruments are Global Positioning System (GPS), Laser Scanner (LS) and Total station (TS).

GPS data were processed using Leica geo-office. GPS coordinates were transformed from World Geodetic System 1984 (WGS84) to local datum. Baselines were processed from four control points to station which is Continuously Operating Reference Station (CORS) that provides Global Navigation Satellite System (GNSS).
[10] in his research thesis titled "Establishing a Geodetic Reference Network in Montserrado County-Liberia, Using GNSS Technology". The aim of the project was to establish geodetic control network in Montserrado Country-Liberia. This was because Liberia's geodetic network was completely destroyed as a result of one and half decades of war experienced. The methodology adopted for the research was the use of satellite positioning technology; Differential Global Positioning System (DGPS) observation.

The result gave huge disparity between the data given and the one generated by means of precise point positioning (PPP). It was a justification that the existing data is not reliable. From the final result, it is concluded that a reliable geodetic reference network is a basic requirement for the successful execution of all survey related projects. It is the wireframe or the skeleton on which continuous and consistent mapping, geographic information system (GIS), and surveys are based. Locating spatial features with respect to geodetic control enables the accuracy assessment of these features. The new network provides coordinates in the World Geodetic System 1984 (WGS84) ellipsoid to be transformed to Liberia local system when transformation parameters are available. It will reduce, if not eliminate compass survey and the metes and bounds method for property location and demarcation as the new network provides reference stations established with sub- centimetre accuracy.

Positioning satellite technologies have been proven to be fast, simple, accurate and cheaper alternative to conventional methods. Tracking signals from Global Navigation Satellite Systems (GNSS) will help in achieving higher accuracies and overcoming numerous limitations associated with terrestrial methods. The method adopted for this research is therefore, that of satellite positioning technology DGPS observations. The static carrier phase 
difference measurement was used for the observation, 3 hours' sessions at each point. MATLAB was used for adjustment computations, Leica Geo-office was used for the initial post processing in Monrovia, and Leica spider Qc and Sokkia spectrum survey were used in Ghana for final post- processing. Data processing underwent two stages; preliminary and final processing. Preliminary processing was done in Liberia immediately after data collection. Field data was initially processed using Leica Geo-office software. The baselines were computed byholding camp Ramrod at $72^{\text {nd }}$ community fixed. This preliminary processing was carried out to judge if the data collected was of quality for the project.

Final processing was carried out using Sokkia spectrum survey software. The base station was fixed by the method of precise point positioning (PPP) with the aid of an online processing service in Canada. Precise ephemeris data was used for the purpose of achieving higher accuracy. An improved set of positions were obtained using least squares minimization procedures and equations modelling potential error sources.

[25]in their work on "Establishment of Geodetic Control in Jebba Dam Using Canadian Spatial Reference System (CSRS-PPP) Processing Software". Canadian Spatial Reference System Precise Point Positioning (CSRS-PPP) uses the so- called point positioning approach (user does not need to be near an Active control points (ACP), International GPS service or continuous reference station (CORS). Unlike differential GPS methods, PPP does not require data from any other GPS receivers [25]. Instead PPP uses precise a priori values of the GPS orbits and of their clocks. These values are obtained from the international GPS service (IGS) and are usually available, in batches of 24 hours, 17 hours, after the end of the GPS day. PPP was used for the establishment of geodetic control in Jebba Dam. The research shows that PPP technique is able to offer mm level accuracy in static mode using the latest IGS satellite orbits and clock production. The main factors that may limit the PPP accuracy are the precision of satellite orbits, clocks and effects of unmodelled errors. The PPP is an efficient and fast way of determining station coordinates. The processing is always online.

[26] in their paper titled "Alternative Ways of Establishing and Protecting Survey Control Points for Successful Implementation of Spatial Enabled Government in Nigeria”. Examined how to preserve survey control points. The control points serve as the major interface or links between the plans and the land area, represented in the plans. This implies the relevance of control points to survey records describing plan or map and test of existence and reliability. The major purpose of survey control points in any operation is to establish a network of control points which will serve as reference stations during surveying. The type of control points employed in surveying can be classified into two categories known as passive control points and active control points [27].

Passive control points: These include all monuments such as pillars, posts, flags, reflectors, etc which can either be physical, occupied or used as targets in the course of surveys. They are often coordinated by conventional survey procedure or satellite observation using Global Positioning System (GPS). These control points are referred to as passive because they cannot communicate and can therefore only be used for surveying, provided they are occupied or employed as dead targets such as in observation with Total stations or Theodolites.

Active control points: These are group of control points that reveal their position either by continuous transmission of identity signals or by responding to interrogation from identifiable signal source. It consists of dual frequency calibrated satellite code and carrier phase observations from continuous 
tracking of GPS satellite. A typical, example of active control point is satellites like GPS.

With the advent of Global Positioning System (GPS) technology, it is imperative that stations can be sighted with greater flexibility with regard to user needs viz ease of accessibility and proximity to settlements. The development of satellite technology, through the use of GPS has opened a new vista in the observation and strengthening of Geodetic Control Networks Worldwide. Most developed countries now use GPS to update their control networks.

[6] examined the Role of African in the Global Geodetic Observing System. The objective of the paper is to review the role of Africa in Global Geodetic Observing System (GGOS). GGOS ensures long term, precise observations of the three fundamental geodetic observables and their variation; earth's shape, gravity field, orientation and rotational parameters, and reference frame, using GNSS, SLRJLLR, VLBI, DORIS and InSAR at Space Geodesy Co-location sites. The objective of the African Geodetic Reference Frame (AFREF) is to establish a unified reference frame through the use of GNSS to tie various national and regional reference networks of Africa. The use of GNSS in Africa involves mostly Global Positioning System (GPS) as of now. Centre for Geodesy and Geodynamics (CGG) at Toro in Bauchi state, Nigeria uses GPS. The main current application of GPS in Africa is for any operation that demands the accurate determination of position or location in terms of geodetic coordinates.

The paper concludes on the importance of establishing centre for Geodesy and Geodynamics (CGG) in other African countries and the extension or densification of the Nigerian centre for Geodesy and Geodynamics to help Africa's role in GGOS with little bit of cooperation. The application of GNSS (GPS) and GGOS are indispensable for numerous human endeavours for national security and socioeconomic developments. That all African countries are expected to promote the realization of AFREF, research and development in space science and technology, and in particular the production of welltrained geodesists.

Geospatial Committee of Arizona Professional Land Surveyors Association in Cooperation with the Arizona Geographic Information Council (2008) in their paper titled "Arizona Spatial Data Accuracy and Geo-referencing"

This paper listed the requirements for geodetic control survey and adjustment.

i. Surveys performed for the purpose of establishing geodetic control shall be based on an appropriately constrained and weighted least- squares adjustment of over- determined and statistically independent observations. If statistically dependent observations are included, their effect on the estimated accuracy must be accounted for and documented.

These are characteristics that apply to a correctly performed survey network adjustment, and they should be part of standard survey practice. They are listed here to make it explicit that correct procedures must be followed, in particular to prevent misrepresentation of a survey as a control survey when it is not. A survey is over determined if it has redundancy (more observations than the minimum necessary to determine positions) which is essential for a meaningful adjustment to be performed.

Statistically independent observations is meant to prevent introduction of false redundancy in a network through representation of the same (or nearly the same) data more than once, which can lead to exaggerated accuracy claims. However, including statistically dependent (trivial) vectors is common practice in Global 
Positioning System (GPS). If trivial vectors are included, the final error estimated should account for the false redundancy created by including these vectors.

It should be noted that considerable professional judgment often comes into play when performing network adjustments, and some allowance should be given for such judgement.

ii. A minimally constrained adjustment must be performed and results documented. The established characteristics of the network must be substantially the same for both the minimally and fully constrained adjustments as determined using standard statistical analyses.

iii. The stations selected for constraining the network shall have coordinates explicitly defined in the datum and realization required for the project, and these coordinates must be documented values obtained from the National Geodetic services (NGS), (or approved equal). Full or weighted constraints may be used but if weighted constraints are used, the values and bases for weighting shall be documented.

The realization of a datum is the reference date that indicates when the adjusted coordinates were determined on a specific datum. In Arizona, for example, NAD 83, has had realizations in 1986 (Original), and 2002 (for the nationwide continuously operating reference station network). Fully constrained control is used when the control coordinates are held absolutely fixed in a network adjustment, making the control in essence "errorless". However, this can cause estimated errors of other points to become too large. One method to reduce this effect is to assign the control standard errors based on estimated accuracy of the control which are used to determine finite weights so that the control is not absolutely fixed. In such cases, the stations used as constrains their coordinates, their assigned standard error, and the datum and realization of the constrained (control) stations should be explicitly listed in the project report.

iv. Local point accuracy estimates determined from the control network shall be based on the fully constrained post- adjustment variance covariance matrix for each point, results shall include at the minimum the horizontal error ellipse axis dimensions scaled to $95 \%$ confidence using the bivariate scalar and the linear vertical error sealed to $95 \%$ using the univariate scalar.

The post- adjustment point variance covariance matrix is a set of numbers that completely defines the estimated accuracy of a point based on the results of a network adjustment. It can be used to compute other quantities, such as the horizontal error ellipse which provide accuracy information in a graphic format. The "Scalars" are numbers used to scale the standard error to a desired confidence level. At the 95\% confidence level the bivariate scalar equals 2.4477 and the univariate scalar equals 1.9600

v. The required final accuracy of an appropriately constrained control survey shall be determined on a projected - by- project basis and specified in the project scope.

vi. Procedures may be less rigorous than the foregoing control survey requirements for establishing control check points within a project area or for establishing points for the purposes of geo-referencing terrestrial (optical) equipment. However, in all cases the method used for establishing such points shall 
be of an accuracy equal to or better than that used for the data collection, and the procedures for establishing such points shall be specified in the project scope. The purpose here is to allow more Freedom for evaluating and supporting certain types of data collection, such as using radial real time kinematic (RTK) GPS to evaluate mapping grade GPS data or using RTK GPS to establish set up and back sight points for an optical total station to collect data in areas where GPS cannot be used.

[2] presented a paper titled "Integrating Nigerian Geodetic Network into the African Geodetic Reference Framework: What are the Issues? The research reviews the need for the many emergence regional and sub- regional cooperation among African countries to establish a unified geodetic reference framework (AFREF) for Africa. This is required in the establishment and maintenance of spatial data infrastructure, mapping, planning of joint projects relating to transportation, boundary demarcation, engineering projects and other spatial data management projects [2]. This would be achieved by each country to establish its own network of Global Positioning System (GPS) control points referred to AFREF (African Reference Frame), which will become part of IGS (International GPS Services) network.

The method to achieve the objective of the research is by carrying out GNSS surveying: Doppler satellite and GPS observations are the methods used in Nigeria. These points must be tied to ITRF (International Terrestrial Reference Frame) which is a global network of points whose 3-dimensional Cartesian coordinates are known and realized from the International Terrestrial Reference System (ITRS). Its realization is based on the estimated coordinates of a set of stations fixed by Very Long Baseline
Interferometry (VLBI), Lunar Laser Ranging (LLR), Solar Laser Ranging (SLR), Doppler Orbitography and Radiopositioning Integrated Satellite (DORIS). The World Geodetic System 1984 (WGS84) reference system is consistent with ITRS and referred to a specified year of its realization. This establishment of a unified geocentric reference framework will involve the estimation of transformation parameters that will be used into transforming local geodetic datum into the world geodetic system.

The conclusion is that the emergence of so many subregional co-operations among African countries calls for the establishment of common reference system. Such a reference framework is required in the establishment and maintenance of spatial data infrastructure, mapping, planning and construction of joint projects relating to transportation, environmental management, boundary demarcation, engineering projects and other spatial data management projects. Each country is expected to establish its own network of points, which will become a part of IGS network. These GPS stations will be used to determine the relationship between the natural datum and ITRF datum, and hence enable the transformation of all spatial information from natural framework to the ITRF, thereby bringing all network points to the ITRF system.

[28] in a paper titled "Establishment of a Precise Geodetic Control Network for Updating the River Nile Maps". The aim of the paper was to establish 600 control stations along the Nile banks at Interval of $5 \mathrm{~km}$. The orthometric height for each control station was also determined alongside with a geoid model for the Nile maps. Also the research, derived standard and specifications being implemented to establish the new national GPS control network. The GPS surveying method was used. The network was tied to some International GPS services (IGS) in order to 
obtain high accuracy estimate. Data processing was carried out in two phases:

1. For a baseline, the followings were performed:

a) Root Mean Square (RMS) error was used to fill in the range from 0.01 to 0.2 cycles

b) The ratio test should be greater than 3

c) The iono-free L1/L2 fixed solution must be obtained for the Nile network

d) Ionospheric and tropospheric model should be implemented

e) Percentage of rejected measurement should be less than $10 \%$

f) Maximum standard deviation of a baseline is 2 $\mathrm{cm}$

g) Tolerance of baseline processing should be better than $2 \mathrm{~cm}$ horizontally and $4 \mathrm{~cm}$ vertically

h) Precise ephemerides are preferable to beutilized rather than broadcasted values.

2. For a session:

a) All combination of baselines in a session should be processed

b) Maximum loop closure in vector components is $25 \mathrm{~cm}$ and

c) Loop closure not exceed $12.5 \mathrm{ppm}$ (12.5 part per million).

The establishment of 600 geodetic control stations to cover both banks of the Nile in an area extends 1435 $\mathrm{km}$ was achieved. Also a set of standards and specifications designed was carefully implemented in order to achieve a high precision national GPS network. The network established with HARN (High Accuracy Reference Network) GPS network will furnish a precise national GPS datum in Egypt.

[12] in a paper titled "The Role of Coordinate Systems, Coordinates and Heights in Horizontal Datum Transformations" highlighted the fundamental definitions of geodetic and geocentric coordinate systems, clarifying the distinction between coordinates and coordinate systems. GPS was used for the establishment of geocentric datum in Australia and the local geodetic datum was transformed to geocentric datum. It is also argued that the horizontal coordinate transformation should not include height information, since this forms an entirely different coordinate in another coordinate system. Which means that transformation of horizontal and vertical coordinates are two different exercises. However, heights are required in the establishment of a geodetic datum to reduce observations from the surface of the earth to the ellipsoid. The satellite geodetic networks are configurations of points whose coordinates have been determined by satellite positioning systems. These positions are inherently three dimensional, and are normally computed in the Cartesian coordinate system. The height obtained by satellite observations are ellipsoid height and to transform them to orthometric (geoid) height, geoid models must be developed.

The conclusion derived from this research is that the transformation should follow two stages: (a) Transformation of the coordinate systems and (b) Modelling of the distortions between the coordinates. These are based on the fact that there is difference between coordinate systems and the coordinates realized by geodetic or geocentric datums. In addition, it has been argued that heights be excluded from a horizontal coordinate transformation, since these comprise an entirely different coordinate system.

[29] in their work on the "Establishment of 3-D Geodetic Control by Interferometry with the TI-4100 GPS Receiver" is aimed at establishing 3-D geodetic control network by using Interferometric type observations of the Global Positioning System (GPS) collected with Texas instruments Tl-4100 GPS receiver. A comparison of the results of a baseline measured with Tl-4100 and V -1000 Interferometric surveyor was carried out. The collected data were 
processed to determine precise 3-D positions of the survey stations without requiring interstation visibility. Five stations located in the vicinity of the study area were occupied during the test. Two caesium clocks aided T1-4100 receivers were employed to obtain simultaneous p-code and carrier phase measurements in two frequencies in a total of 13 sessions. The evaluation and analysis of the data was focused on the operational aspects, data processing techniques and accuracy of the estimated positions.

GPS satellite orbits modelling was carried out in the research. T1-4100 receivers can decode the GPS signal, the orbital information needed for processing the collected data is obtained by occasional dumps (typically every hour) of the broadcast ephemeris reports. These broadcast ephemerides are based on 24 hourly predictions from historical and near-real time tracking by the US DoD.

T1-4100 receivers can give one of the observables as one-way phase of the signal received from each of up to four satellites each with respect to a reference signal that is derived from the receiver oscillator. Comparison of short- Arch orbits computed from truncated Gen-9 potential model and $9^{\text {th }}$ order. Tehebychev polynomial approximations with GPS broadcast ephemeris was carried out.

The results achieved from the Tl-4100 test obtained agreed very well with the terrestrial coordinate standard. The consistency of the differences in baseline lengths and azimuths between the GPS and terrestrial-derived positions indicate very clearly that the coordinates of one system can be adjusted to be compatible with the other system to within a few tenths of an arcsecon in orientation and better than 2 ppm in scale. The results demonstrated that, positioning accuracies of 1-2 $\mathrm{ppm}$ are feasible for distances between 10 and $100 \mathrm{~km}$. There is no question that this new technology will produce a major redirection of positioning methodology, accelerating the development that started with TRANSIT Doppler and Inertial positioning methods. [30] in his paper titled "Global Positioning System (GPS) Control for Digital Mapping" considered the use of GPS with Photogrammetry in terms of their spatial configuration and surveying techniques for the establishment of GPS control for digital mapping:' GPS was used for the establishment of ground controls for photogrammetry mapping. In photogrammetry what looks like the central receiver is the aerial camera.

The aerial base vector (b) is determined by the relative and absolute camera positions in two exposure moments. Two ground located GPS receivers are in a similar mutual position. The socalled position (or base) vector (d) is the result of the relative and absolute position fix of the two GPS receivers.

The disparity between GPS and Photogrammetry is that in photogrammetry, the control points are located on the ground, and the base vector is in the sky. In GPS, inversely, the base vector is situated on the ground and the control points -the satellite are located "in the sky". In both systems there is a relative motion between the 'central receiver and control point'. (As forward motion in photogrammetry, the consequent Doppler effect is also compensated for in GPS). The requirement of an open sky stays relevant as a trivial demand both for photo and GPS techniques. In height, the GPS generally is slightly less accurate than in horizontal position, mainly because of the uncertainties of geoid undulations. However, connecting with precisely levelled benchmarks and applying an appropriate and simple estimate for undulations even in large 
photogrammetric control networks, the absolute height accuracy will remain in the range of centimetres.

The result is that GPS is the best tool today for geodetic control of digital photogrammetric mapping. It is full digital in nature, efficient, sure, rapid, accurate and relatively inexpensive.

\section{SUMMARY/GAPS IDENTIFIED FROM LITERATURES REVIEWED}

From the literatures reviewed, the similarities observed by the works of the following researchers; [24], [10], [25] and [28] is that they all used precise ephemerides for final data analysis. However, each of the researchers used different methods of Global Positioning System (GPS) surveying; These methods are stated as follows:

[24] was to evaluate coordinates obtained by GPS, Total station and Terrestrial Laser scanner and compare their precision, accuracy and Time expenditure. The Global Positioning System (GPS) surveying was carried out and data processing used precise ephemerides for final analysis. Although broadcast corrections were applied by a base receiver to solve for coordinates at a rover receiver in real time kinematic. GPS data were processed using Leica Geo-office.

[10] used GPS to establish geodetic control network in Montserrado County-Liberia. To be specific, Differential Global Positioning System (DGPS) observations were carried out. At a point, the researcher used precise point positioning (PPP) and compared the result with the given coordinates, but a huge disparity was discovered. This was a justification that the existing coordinates were not reliable. The static carrier phase difference measurement was used for the observation, three (3) hours session at each point. Mat-Lab was used for adjustment computations. Leica Geo-office was used for the initial post processing and Leica Spider QC and Sokkia spectrum survey software were used for final post processing. Here, data processing underwent two stages, namely, preliminary data processing was carried out to judge the quality of data collected whether they meet the requirements of the project.

Final processing was carried out using Sokkia spectrum survey software. The base station was fixed by the method of precise point positioning (PPP) with the aid of online processing service in Canada. PPP uses precise ephemeris and not broadcast ephemerides. This makes the final processing similar to that of [24].

[25] used Canadian Spatial Reference System (CSRSPPP) processing software in the establishment of Geodetic Control in Jebba Dam. This uses precise point positioning method (user does not need to be near an active control points (ACP). PPP method is not like the Differential Global Positioning System (DGPS) methods. PPP does not require data from any other GPS receivers. Instead, PPP uses precise point values of the OPS orbits and of their clocks. These values are obtained from the International GPS services (IOS) and are usually available, in batches of 24 hours, 17 hours, after the end of the OPS day. It was shown that PPP technique is able to offer millimetre $(\mathrm{mm})$ level accuracy in static mode using latest IGS satellite orbits and clock production. The main factors that may limit the accuracy of PPP are the precision of satellite orbits, clocks and effect of un-model errors. The final processing is always online using precise ephemerides. This makes it similar with the final processing carried out by [24], [10]. 
[26] discussed on the importance of using GPS for the establishment of geodetic control networks. The paper also stated the advantages that the advent of GPS has brought to surveying as a profession and the need to install monuments to define survey points and their protection. The need for Nigeria to update her geodetic control networks to the World Geodetic System 1984 (WGS84) ellipsoid. These researchers only talked about the theoretical aspect of establishing geodetic control network.

[6] discussed the role of Nigeria in having a unified geodetic datum for Africa. The objective the African Geodetic Reference Frame (AFREF) is to densify Africa with Global Geodetic Observing System (GGOS), such as the Continuously Operating Reference Stations which are based on WGS84 ellipsoid. GPS is based on WGS 84, therefore, for an effective use of GPS in Africa, WGS84 ellipsoid must be considered. Individual country's local datum needs to be transformed to WGS84.

[2] also discussed the importance of integrating Nigerian Geodetic Network into the African Geodetic Reference Frame (AFREF). He observed that the use of GPS for the establishment of geodetic control network is very important. GPS established geodetic controls are always based on the global datum. Therefore, transformation from one datum to another will be necessary where local datum has been in existence. The importance of having a unified reference datum for Africa is for the establishment and maintenance of spatial data infrastructure, mapping, planning and construction of joint projects relating to transportation, environmental management, boundary demarcation, engineering projects and other spatial data management projects.

Arizona Spatial Data Accuracy and Geo-referencing (Geospatial Committee of the Arizona Professional Land Surveyors Association in Cooperation with the
Arizona Geographic Information Council (2008), listed the requirements for the establishment of geodetic control network: (1) purpose for establishing geodetic control, (2) adjustment constraints, (3) station selection for the network, (4) local point accuracy procedures, and (5) final accuracy. No practical work was carried out, therefore, the paper limited its finding to theoretical requirements.

[12], as well as [2], defined the fundamental difference between geodetic and geocentric coordinate systems, distinction between coordinates and coordinate system. GPS was used for the establishment of geocentric datum in Australia. The established control networks were on a different ellipsoid to that of local datum. It is therefore needful to bring the two ellipsoids together, which is the required transformation. This is found lacking in the works of the aforementioned authors. Therefore, to correct this anomaly, the horizontal coordinate transformation will be treated different from the vertical control because the two are not on the same datum. GPS always gives direct three-dimensional coordinates, but the vertical height is ellipsoidal. Therefore, the height obtained by GPS is not orthometric height. Orthometric height is reference to the geoid and to convert ellipsoidal height to orthometric height, geoid undulation must be determined to develop geoid model.

[29], discussed the establishment of 3-D geodetic control by Interferometry with Tl-4100 GPS receiver. They maintained that this type of GPS receiver is far less sensitive to orbital uncertainties than point positioning baseline determinations using the carrier phase observations, which in itself is limited in accuracy by orbit errors. They also stated that the problem with the broadcast orbits, though seemingly adequate for a few parts per million (ppm) relative positioning, should only be used for the one hour for 
which they are intended. Extrapolation beyond that point leads to an exponential growth of truncation error. Even if no extrapolation is attempted, satellite positions for the same epoch determined from two adjacent broadcast messages tend to show significant discontinuities of the order of several metres which can severely bias the final results. In particular, a distorted orbital shape can affect the relative positioning accuracy especially for large station separation and a short time span of data. The paper went further to make a comparative analysis of the short-arc orbit with that of broadcast orbit and stated some of the implications of using broadcast orbit. However, gap still remain that other orbital data were not analysed.

[30] discussed the relationship between GPS and photogrammetry. The importance of using GPS to establish ground point rather than photogrammetric observations. Though photogrammetry is expensive, it can be combined with GPS for the establishment of geodetic control. This is because in height determination, GPS generally is slightly less accurate than in horizontal positions mainly because of the uncertainties of geoid undulations. However, connecting with precisely levelled benchmarks and applying an appropriate and simple estimate for undulations even in large photogrammetric control networks, the absolute height accuracy will remain in the range of centimetres. It therefore remained that GPS is the best tool for geodetic control of digital photogrammetric mapping because it is rapid, accurate, efficient, sure, and relatively inexpensive. This research is silent on GPS data processing.

\section{REFERENCES}

[1]. C. D. Ghilani and P. R. Wolf, Elementary Surveying, An Introduction to Geomatics, USA: Pearson Prentice Hill Educational international, 2008.

[2]. C. U. Ezeigbo, "Integrating Nigerian Geodetic Network into the African Geodetic Reference Framework: What are Issues?," in Nigerian Institution of Surveyors (NIS) 39th AGM and Conference, Port-Harcort, Nigeria, 2004.

[3]. A. Leick, GPS Satellite Surveying, London: John Wiley and Sons, 2012.

[4]. B. F. Kavanagh, Surveying with Construction Application, Prentice Hall, Boston: Pearson Education Inc, 2010.

[5]. C. Jikeli, Geometric Reference Systems in Geodesy, Division of Geodesy and Geospatial Science, School of Earth Science, Ohia State university, 2006.

[6]. D. M. J. Fubara, F. A. Fajemirokun and C. U. Ezigbo, Fundamental of Geodesy, Lagos: Concept Publication (Press Division), 2014.

[7]. N. V. Uzodinma, J. O. Oguntuase, N. O. Alohan and C. N. Dimgba, Practical GNSS Surveying, Enugu: Professors' Press Limited, 2013.

[8]. E. C. Onyeka, Geoinformatics in Environmental Monitoring, Enugu: Sac Press Ltd, 2007.

[9]. P. F. Dale and J. D. McLaughin, Land Information Management. An Introduction with Special Reference to Cadstral Problem in Third World Countries., New York: Claredon Press Oxford, 1990.

[10]. C. S. Bundoo, "Establishing a geodetic Reference Network in Montserrado County, Liberia using GNSS technology," Unpublishe MSC Thesis, Kwame Nkruma Univeristy of Scienece and Tehnology (KNUST), Kumasi, Ghana, Kumasi, Ghana, 2013. 
[11]. ASCO, "Geodetic," 2018.

[12]. W. Featherstone and P. Vanicek, "The Role of Coordinate Systems, Coordinates and Heights in Horizontal Datum Transformation.," The Australian Surveyors. 44(2) FGGC, 1999.

[13]. JPO, "NAVSTAR GPS User Segment," NAVSTAR -GPS Joint Program Office, 1991.

[14]. A. Leick, "Accuracy Standards for Modern Three-Dimensional Geodetic Networks," ACSM Surveying and Land Information System. 53(2), pp. 111-116, 1992.

[15]. CGCC, "Califonia Geodetic Control Committee,Specifications for Geodetic Control Network Using High-Producction GPS Surveying Techniques, Version 2.0," 20 March 2018. [Online]. Available: http://www.rbf.com/cgcc/hpgps21.htm.

[16]. S. O. Eteje, "Comparative Analysis of Geodetic Techniques for Monitoring Deformation in large Structure," Unpublished $\mathrm{PhD}$ Disseration of the Department of Surveying and Geoinformatics, Nnamdi Azikiwe University, Awka, Anambra state Nigeria, 2019.

[17]. A. Leick, GPS Satellite Surveying, London: John Wiley and Sons, 1990.

[18]. E. M. Mikhail and G. Gracie, Analysis and Adjustment of Survey Measurements, New York: Van Nostrand Reinhold Company, 1981.

[19]. W. J. Ghitumbi, "Deformation Monitoring of an Oil Storage Tank by Geodetic Methods; A Case Study of Oil Libya, Nairobi Terminal," Department of Geospatial and Space Technology, University of Nairobi, Nairobi, 2014.

[20]. B. M. Ameh, "Determination of Component of Deflection of Vertical of Lobi Area of Makurdi, Benue State Using GPS and Precise Levelling," Unpublished MSC Thesis of the Departmrnt of Surveying and Geoinformatics, Nnamdi
Azikiwe University, Awka, Awka, Anambra state, 2013.

[21]. W. F. Caspary, Concept of Network and Deformation Analysis, New South Wales, Kensington, Australia: School of Surveying, University of New South Wales, Australia. ISBN 0-85839-044-2, 1988.

[22]. L. L. Arinola, "Error propagation in the Nigerian geodetic network: imperatives of GPS observations to strengthen network.," in XXIII International FIG Congress, Munich Germany, 2006.

[23]. O. Onabajo, "History of Nigeria Geodetic Network Summarized," OSGOF Journal, 2006.

[24]. S. D. Chekole, "Surveying with GPS, Total Station and Terrestrial Laser Scanner; A Comparative Study," Unpublished Thesis, tritaGit Ex14-001 School of Architecture and theBuilt Environment, Royal Institute of Technology, Stockholm, Sweden, 2014.

[25]. R. E. Irugh, M. O. Elugiator and S. A. Uzoekwe, "Establishment of Geodetic Controls in Jebba Dam using CSRS-PPP Processing Software.," Jornal of Emerging Trends in Engineering and Applied Sciences, 2(5), pp. 763 - 769, 2011.

[26]. J. T. Fadahunsi and A. K. Tella, "Alternative ways of Establishing and Protecting Survey Control Point for Successful Implementation of Spatial Enabled Governance in Nigeria," in Nigerian Institution of Surveyors 45th AGM and Conference, Abuja, 2010.

[27]. O. C. Ojinaka, Principles of Hydrographic Surveying from Sextant to Satellite, Enugu: El Demak, 2007.

[28]. G. M. Dawod and T. M. Abdel-Aziz, "Establishment of a Precise Geodetic Control Network for Updating River Nile Maps," in Proceeding of AlAzhar Engineering. 7th International conference, Cairo, 2003. 
[29]. D. Delikaraoglou, N. Beck, D. McArthur and K. Lochhead, "On Establishment of 3-D Geodetic Control by Interferometry with the T1-4100 GPS Receiver," Geodetic Survey Division, Surveys and Mapping Branch, Energy, Mines and Resources, 1985.

[30]. J. Forrai, "Global Positioning System for Digital Mapping," in ISPRS Commission IV, 2009.

\section{Cite this article as :}

Ikharo I. Blessing, Matthew N. Ono, Olaniyi Saheed S., "Establishment of Geodetic Network in Nigeria", International Journal of Scientific Research in Science and Technology (IJSRST), Online ISSN : 2395-602X, Print ISSN : 2395-6011, Volume 6 Issue 4, pp. 197-221, July-August 2019. Available at doi : https://doi.org/10.32628/IJSRST196436 Journal URL : http://ijsrst.com/IJSRST196436 